\title{
Emery-Dreifuss muscular dystrophy: a test case for precision medicine
}

\author{
This article was published in the following Dove Press journal: \\ The Application of Clinical Genetics \\ 24 February 2016 \\ Number of times this article has been viewed
}

\author{
De-Ann M Pillers' \\ Nicholas HVon Bergen ${ }^{2}$ \\ 'Division of Neonatology and \\ Newborn Medicine, ${ }^{2}$ Division of \\ Cardiology, Department of Pediatrics, \\ University of Wisconsin-Madison, \\ Madison, WI, USA
}

\begin{abstract}
Emery-Dreifuss muscular dystrophy (EDMD) is characterized by the clinical triad of scapulohumeroperoneal muscle weakness, joint contractures, and cardiac defects that include arrhythmias and dilated cardiomyopathy. Although there is a defining group of clinical findings, the proteins responsible and their underlying gene defects leading to EDMD are varied. A common aspect of the gene defects is their involvement in, or with, the nuclear envelope. Treatment approaches are largely based on clinical symptoms. The genetic diversity of EDMD predicts that a cure will ultimately depend upon the individual's defect at the gene level, making this an ideal candidate for a precision medicine approach.
\end{abstract}

Keywords: emerin, FHL1, lamins A/C, nuclear envelope

\section{Introduction}

\section{Lumpers and splitters}

Throughout the history of medicine and science, the concepts of "lumping and splitting" have been used to identify, characterize, understand, and translate knowledge about disease into therapy. Hence, these concepts have been used for the study of EmeryDreifuss muscular dystrophy (EDMD). Originally lumped with other X-linked muscular dystrophies, most notably the Duchenne muscular dystrophy (DMD) and Becker muscular dystrophy (BMD) forms, EDMD has, via careful patient evaluation, new data and astute clinicians split off to become a discrete entity. Now, the new "lump" of EDMD has undergone its own splitting as alternate inheritance mechanisms have suggested alternate etiologies and genetic defects with the same clinical findings. Inasmuch as EDMD has split, it is now being lumped again with what was previously considered to be a separate disorder: rigid spine syndrome. Rigid spine syndrome may simply be a phenotypic variant of EDMD. It is becoming clear that there are modifying effects independent of the gene defect that have resulted in patients with EDMD with a wide array of physical findings, albeit all are based on the clinical triad of joint contractures, scapulohumeroperoneal muscle weakness, and cardiac arrhythmias. ${ }^{1}$ These various permutations and combinations of EDMD suggest that it may be a test case for "precision medicine", a concept introduced by the administration of United States President Barack Obama in January, 2015, as a path to individualized therapy based upon a person's genetic make-up and environmental modifiers. This review will begin with the history of EDMD and its key players, from its initial description through the evolution of knowledge relating to its underlying defects, and end with a vision of therapy for the future and how precision medicine may play a role in accomplishing that. 


\section{A history of EDMD}

The original description of EDMD has generally been attributed to Dreifuss and Hogan. ${ }^{2}$ Fred (né Fritz) Dreifuss was a highly lauded neurologist whose passion was epileptology, and his particular emphasis for study was the absence seizure. $^{3} \mathrm{He}$ is credited with the development of the continuous electroencephalogram as a clinical tool for confirming a diagnosis of epilepsy. He applied this tool to monitor the success of drug trials for absence seizures and was a pioneer in the use of sodium valproate for this disorder. Born in Dresden (1926), he escaped from Germany in the 1930s to New Zealand via South Africa, and after training in New Zealand and the United Kingdom, he moved to Charlottesville, VA, where he headed up the Child Neurology program at the University of Virginia, and there he remained until his death in 1997.

Outside of his internationally acclaimed university-based academic practice, Dreifuss routinely provided care via outreach clinics to the Appalachian communities of Virginia. The discovery of EDMD came as a result of these outreach clinics with the description by Dreifuss and Hogan of a family of English descent living in the coal mining Appalachian plateau area of Buchanan County, who had muscle wasting disease that was originally believed to be a variant of BMD. ${ }^{2}$ The recognition that this family represented a novel form of muscular dystrophy came in 1966, via a publication by Dreifuss with Alan EH Emery. ${ }^{4}$ Emery has been a prolific contributor to the clinical understanding of muscular dystrophies and their genetic basis and has published numerous books, papers, and monographs on the subject. He is a native of the United Kingdom who was based at Manchester University at the time of publication of the Emery-Dreifuss report. Emery credits his scientific interests to his background in botany and his clinical interests in muscle diseases and ability to relate to patients due to his childhood experience with osteomyelitis, which resulted in his need to wear "callipers" (ie, leg braces) as a child. ${ }^{5}$

The original three-generation EDMD family included eight affected males and 16 "unaffected" carrier females., ${ }^{2,4}$ The disease course was described as "remarkably uniform" and was characterized by the onset of proximal muscle weakness during childhood that first affected the lower extremities in association with flexion contractures of the elbows and shortening of the Achilles tendon, resulting in the development of "toe walking". Affected adults manifested a lordotic stance, a waddling gait, and absence of the deep tendon reflexes. The third characteristic finding of EDMD was also noted in this family and consisted of arrhythmias, ranging from a junctional rhythm likely as a result of the absence of atrial activity (atrial standstill) to atrial fibrillation and even sudden cardiac death.

The serum creatine kinase (CK) levels were elevated in the affected family members, although not to the same degree as occurred in DMD or BMD. Other key distinctions from DMD/BMD were the absence of developmental delay and a lack of pseudohypertrophy of the calf. Thus, the entity now known as X-linked EDMD was confirmed as a unique clinical syndrome in $1966 .{ }^{4}$ The misconception that female carriers are unaffected has since been rectified using the original family and another three-generation pedigree, as we now know that six of the obligate carriers (18\%) manifested cardiac arrhythmias, including two carriers who had pacemakers. ${ }^{6}$ Three major groups of proteins have been associated with EDMD: emerin, FHL1, and lamins A/C. ${ }^{7,8}$ Their shared location at the nuclear membrane has made them part of the group of disorders known as "nuclear envelopathies".

\section{Clinical features and treatment}

The typical age of onset of EDMD is during adolescence, but it can be present in childhood, especially in families where others are affected and symptoms and findings are more easily understood and recognized. ${ }^{7}$ A slowly progressive proximal muscle weakness followed by joint contractures leading to tightness of the muscle groups heralds the onset of EDMD-specific symptoms. Toe walking due to tightening of the Achilles tendon is another common presentation. ${ }^{1}$ The muscle weakness is in a scapulohumeroperoneal distribution that eventually leads to the involvement of the scapular and pelvic girdle muscles and results in the classic findings of winged scapulae and pelvic tilt. ${ }^{7}$ There may also be tightening of the spinal extensor muscles, causing limitation in the mobility of the neck and spine. ${ }^{1}$ In some cases, the decreased mobility of the spine is thought to contribute to respiratory disorders, including hypoventilation due to a restrictive pattern of respiratory impairment. ${ }^{10}$ Carriers are not usually affected by the musculoskeletal manifestations of EDMD.

Treatment of the neuromuscular aspects of EDMD is largely based on addressing the symptoms. ${ }^{7}$ When EDMD symptoms are recognized early, physical therapy and stretching are used to minimize contractures. Ambulation can be assisted with walkers and canes, and when the symptoms progress, a wheelchair may be needed. Surgery can also be done to release contractures once they have developed and to manage scoliosis. Surgical interventions carry a risk for rhabdomyolysis and severe hyperkalemia, and so judicious choices by the anesthesiologist in the use of muscle 
relaxants and inhaled anesthetics are required. ${ }^{11}$ The final clinical finding of the triad described in the original report by Emery and Dreifuss in 1966 is that of cardiac disease. ${ }^{4}$ Bialer et al, ${ }^{6}$ also based at the University of Virginia, Charlottesville, have provided key insights into this aspect of the disease and noted that there may be a "disconnect" between the degree of the muscle and the cardiac disease. Although the skeletal muscle disease may be benign compared to other muscular dystrophies, the cardiac phenotype can be severe, even leading to sudden death. EDMD typically does not have clinically significant cardiac findings until the affected individual reaches their second or third decade of life, with the incidence of electrocardiogram (ECG) abnormalities increasing with age. ${ }^{6}$ Manifesting carrier females may be much older but are also at risk of sudden death as a result of their rhythm disturbances. ${ }^{6,12}$

Patients with EDMD may develop tachycardia such as atrial tachycardia, atrial fibrillation or flutter, and ventricular arrhythmias or may develop bradycardia such as sinus bradycardia, junctional rhythm, or various degrees of heart block as a result of conduction tissue disease. ${ }^{6}$ The initial cardiac finding in EDMD is often a first-degree atrioventricular block, which occurs when there is slowing of the electrical conduction system of the heart through the atrium or the AV node and results in a prolongation of the PR interval on the ECG. Patients may manifest both a prolonged P-wave duration and an increased $\mathrm{P}$-wave dispersion that is a measure of the difference between the shortest and longest $\mathrm{P}$-wave durations recorded from different leads and serves as a marker for the risk of paroxysmal atrial fibrillation. ${ }^{13}$ In and of itself, first-degree heart block is not a significant clinical problem, and most patients are asymptomatic. However, this may be an indication of the onset of progressive disease. One of the classic findings in EDMD is atrial standstill, which often presents as bradycardia with a junctional or an idioventricular escape rhythm as a result of the absence of electrical activity in the atrium. Patients who develop second- or third-degree heart block will show variable or complete loss of signal transmission from the atrium to the ventricle via the AV node. These more serious rhythm abnormalities may be associated with significant symptoms such as fatigue, syncope, and even sudden cardiac death. ${ }^{6}$ As heart disease may be progressive, it is imperative to screen potential patients with EDMD for evidence of cardiac involvement. Tachycardia may require management, and when patients have worsening bradycardia or signs of conduction tissue disease, a cardiac pacemaker may be lifesaving. ${ }^{14} \mathrm{~A}$ screening ECG is the test commonly used to diagnose rhythm disturbances in EDMD.
However, because it has been shown that some clinically asymptomatic individuals have serious disturbances at night when they are asleep, an ECG alone may not be sufficient. Therefore, a continuous 24-hour ECG monitoring test should be considered. ${ }^{6,15,16}$

Implantation of a cardiac pacemaker when symptomatic bradycardia or conduction disease is identified may be essential to drive the ventricle at an appropriate rate, which can decrease the risk of potentially life-threatening arrhythmias, such as ventricular tachycardia. ${ }^{14,17}$ Unfortunately, a pacemaker does not eliminate the cardiac risks of EDMD as there are many reports of such patients succumbing to sudden cardiac death even after device implantation. Whether this is due to pacemaker failure or to the other cardiac manifestations of EDMD is unclear. ${ }^{6}$ Congestive heart failure may result from the development of cardiomyopathy, typically a later finding in EDMD, which may be due to progressive myocardial fibrosis or cardiac myocyte disarray leading to ventricular dilation and diminished function. When patients are paced, they also become at risk for the sequelae and the associated rhythm disturbances that can occur in paced hearts, such as pacemaker-mediated cardiomyopathy. ${ }^{6,18}$

\section{Diagnostic findings}

Establishing the diagnosis of EDMD is tied to the clinical triad of joint contractures, proximal muscle weakness, and cardiac disease with conduction defects and rhythm abnormalities. ${ }^{19}$ To support the clinical diagnosis, tests that can be pursued include electrocardiography and echocardiography, which may show the rhythm abnormalities or cardiomyopathy described earlier. Although electromyography can be used in differentiating between some muscle disorders, there is no classic electromyography finding in EDMD. ${ }^{19}$ Computed tomography scans of the muscle typically show the diffuse involvement of the muscle in the scapulohumeroperoneal distribution. ${ }^{19}$

Emerin is ubiquitously expressed at the nuclear membrane and can be assayed without muscle biopsy by using exfoliated buccal cells, skin biopsy, or blood samples..$^{20,21}$ When a muscle biopsy is required, immunocytochemistry may be informative if defects are found in emerin and FHL1, but immunocytochemistry and immunoblots have not proven to be useful in establishing a diagnosis with defects in the lamins $\mathrm{A} / \mathrm{C}$ because the redundancy in the isoform expression with overlaps in function results in a normal appearing study. ${ }^{22}$ FHL1 is ubiquitously expressed in the cytoplasm and nucleus and can be assayed using muscle tissue, cardiac 
muscle, and fibroblasts. ${ }^{23}$ Lamins $\mathrm{A} / \mathrm{C}$ are expressed at the nuclear membrane and in the nuclear matrix. ${ }^{22}$

CK levels may be elevated by $2-20$-fold. ${ }^{7}$ When an elevated $\mathrm{CK}$ is found, it is appropriate to pursue genetic testing to establish the diagnosis. Next-generation sequencing and gene panels are commonly used for this purpose. ${ }^{24}$

\section{“Splitting EMD" according to inheritance pattern}

EDMD occurs in X-linked and autosomal forms with multiple subtypes now described with varying gene mutations. The spectrum of diseases that are allelic variants to EDMD at these gene loci is rapidly expanding in large part due to the recent discovery that EDMD is associated with abnormalities in the nuclear envelope (NE) proteins. The most common form of EDMD is X-linked recessive, and it is associated with defects in emerin, named for Alan Emery, and is encoded by the Emerin, alternately known as STA or EMD gene. ${ }^{25}$ Lamins $\mathrm{A}$ and $\mathrm{C}$ are encoded by the $L M N A$ gene and are autosomal in their inheritance pattern, with most being autosomal dominant. ${ }^{22}$ Mutations in the $L M N A$ gene are not exclusive to EDMD as they have also been associated with a form of congenital muscular dystrophy and with limb-girdle muscular dystrophy type $1 \mathrm{~B} .{ }^{26} \mathrm{FHL} 1$ is the most recent gene to be associated with the EDMD phenotype and, like EMD, has an X-linked recessive inheritance pattern. ${ }^{23}$

Although these various forms of EDMD and its closely allied disorders overlap in their clinical spectrum and are currently managed with standard medical and surgical interventions, it is clear that any ability to provide affected individuals with a cure will be tied to knowing the correct molecular diagnosis, consistent with the precision medicine approach. Although the precision medicine initiative is targeted to cancer therapies, EDMD would also appear to be an ideal test case for this new initiative based on the diversity of disease mechanisms involved. Prenatal diagnosis for EDMD is also facilitated by knowing the underlying gene defect. ${ }^{19,27}$

\section{Genetic diversity in the pathogenesis of EDMD}

The common triad of findings in EDMD belies the reality that there are multiple different genes that can be involved. Understanding the genotype in the context of the phenotype can be critical in developing new therapeutic approaches for a disorder for which there is, at present, no cure. Although there are at least seven genes that have been identified as causes of EDMD and multiple non-disease-causing modifier genes, the three most common causes of EDMD remain EMD, LMNA, and $F H 1$, which are discussed later.

\section{Emerin}

Nearly two-thirds of the patients with EDMD with an X-linked inheritance pattern have their defect in emerin (MIM 300383; EMD type 1). ${ }^{19,28}$ Emerin is a single-membrane spanning protein that localizes to the inner nuclear membrane and is ubiquitously expressed. ${ }^{29}$ The gene is located at Xq28 and is encoded by the $S T A$ or EMD gene. ${ }^{30}$ In muscle, an emerinnuclear protein complex has been proposed to be located at the NE with a key function of the complex to stabilize the nuclear membrane during the mechanical stress that is generated with muscle contraction. ${ }^{29}$ The genotype-phenotype correlations for defects in emerin and EDMD are like those of dystrophin and DMD. Dystrophin similarly forms the basis of a protein-binding complex. The muscle disorder is X-linked recessive in both instances, and there may be manifesting female carriers. EDMD is also like DMD in that the absence of protein expression due to a null mutation results in a more severe phenotype than if the mutation results in a truncated protein. ${ }^{31,32}$ In the milder phenotype, the mutant protein is directed to the inner nuclear membrane. ${ }^{32}$

\section{Lamins A/C}

Mutations in $L M N A$ are responsible for $\sim 45 \%$ of cases of autosomal dominant EDMD (MIM 181350; EMD type 2). ${ }^{19,28}$ $L M N A$ is located at $1 \mathrm{q} 11-\mathrm{q} 23$, and the inheritance pattern is usually autosomal dominant, although autosomal recessive inheritance has also been described (MIM 181350; EMD type 3$).{ }^{26,28}$ It encodes the lamins $\mathrm{A}$ and $\mathrm{C}$, which, like other lamins, are components of the $\mathrm{NE}$ and are located in the lamina. ${ }^{26}$ No clear genotype-phenotype correlation has been shown with $L M N A$ mutations, likely because of the redundancy of the protein loops that can overlap with each other to sustain function. Although the clinical features overlap between EMD1 and EMD2, EMD2-affected individuals are more likely to have loss of ambulation. ${ }^{33}$ Mutations in $L M N A$ have been associated with a variety of phenotypes, including limb-girdle muscular dystrophy type 1B, dilated cardiomyopathy with conduction defects, autosomal recessive axonal neuropathy (CMT2B1), and Hutchinson-Gilford progeria syndrome. ${ }^{33}$

\section{FHLI}

Mutations in FHL1 are responsible for $\sim 10 \%$ of EDMD with an X-linked inheritance pattern. The FHL1 gene is located at Xq26.3 (MIM 300163; EMD type 6). ${ }^{19,28}$ Three different 
isoforms are usually expressed by this gene. ${ }^{19}$ Each isoform expresses proteins that have "Four and a Half Lim" domains. LIM proteins contain two zinc finger proteins in tandem that act as sites to interact with other proteins, and the cysteine residues are particularly important sites for mutation because they disrupt the zinc finger activity. ${ }^{19}$ Mutations in FHL1 are associated with considerable variability in phenotype, with a given mutation being described in multiple clinical syndromes. The expression of FHL1 in female carriers is also variable. Studies evaluating muscle cells derived from patients with EDMD suggest that FHL1 mutations lead to altered myotube formation. ${ }^{23}$

Less common causes of EDMD are autosomal dominant EMD4 (MIM 612998), which is due to defects in nesprin-1 encoded by the SYNE1 gene, and autosomal dominant EMD5 (MIM 612999), which is due to defects in nesprin-2 encoded by the SYNE2 gene. ${ }^{28}$ Nesprin-1 and -2 are members of the NE spectrin-repeat (nesprin) family that act to tether the outer membrane of the NE to the cytoskeleton via binding to F-actin. ${ }^{34}$ Variants in two additional proteins in the LINC (linker of nucleoskeleton and cytoskeleton) complex at the nuclear envelope, SUN1 and SUN2, have also been described in association with the clinical phenotype of EDMD in patients without mutations in other genes. ${ }^{35,36}$ LUMA is a nuclear membrane protein that is a binding partner for both SUN2 and emerin and is encoded by the gene TMEM43. ${ }^{37}$ Mutations in TMEM43 are also associated with an EDMDrelated myopathy (EDMD7). ${ }^{37}$ Digenic inheritance in which mutations in two different EDMD genes are found has also been described. ${ }^{38,39}$

\section{Precision medicine}

The Precision Medicine Initiative was developed by the Obama administration and unveiled during his 2015 State of the Union address to denote a personalized approach to therapy based on an individual's mutation and response to treatment. ${ }^{27}$ Although the initiative is primarily focused on cancers with a genetic basis, the concept is far reaching, and there is no better example than EDMD of why it is important to move away from the "one size fits all" approach to therapy.

The unifying feature of the group of disorders known as EDMD is a triad of clinical findings: contractures, scapulohumeroperoneal muscle weakness, and cardiac disease. These findings are a common endpoint but are arrived at by traveling very different paths depending on the gene that is mutated. Each gene encodes a protein that has very discrete functions, which interact in some fashion with the
$\mathrm{NE}$ to result in a final common pathway to generate the phenotype. How that occurs is an individual event as each gene is associated with considerable diversity in its manifestations. At present, there is no cure for any of the subtypes of EDMD, and a search at ClinicalTrials.gov reveals no clinical research protocols that have reached the patient enrollment stage.$^{40}$ Current therapies are aimed at the clinical symptoms and are largely supportive. For example, identification of individuals at risk for sudden cardiac death may lead to the potentially lifesaving intervention of cardiac pacer placement but does not actually eliminate the risk of sudden death or cure the cardiac disease. Therefore, screening of family members, both genetically and clinically, continues to be especially important to help identify family members who may be at risk. Developing novel therapies that are based on an individual's genetic complement may be one path by which EDMD finally has a "cure". Thus, understanding the diversity of genes and mutations involved is an important adjunct both in a precise diagnosis and in developing future therapies for EDMD.

\section{Acknowledgment}

The authors thank Dr David Hsu, Pediatric Neurology at the University of Wisconsin-Madison, for helpful review of the manuscript.

\section{Disclosure}

The authors report no conflicts of interest in this work.

\section{References}

1. Dubowitz V. Muscle Disorders in Childhood. 2nd ed. Philadelphia: W.B. Saunders Co, Ltd; 1995.

2. Dreifuss FE, Hogan GR. Survival in X-chromosomal muscular dystrophy. Neurology. 1961;11:734-737

3. Porter RJ. In Memoriam: Fritz E. Dreifuss 1926-1977. Epilepsia. 1998;39:556-559.

4. Emery AEH, Dreifuss FE. Unusual type of benign X-linked muscular dystrophy. J Neurol Neurosurg Psychiatry. 1966;29:338-342.

5. Anonymous. The dissecting room: Alan E.H. Emery. Lancet. 2000;355:1566.

6. Bialer MG, McDaniel NL, Kelly TE. Progression of cardiac disease in Emery-Dreifuss muscular dystrophy. Clin Cardiol. 1991;14:411-416.

7. Bonne G, Capeau J, De Visser M, et al. 82nd ENMC international workshop, 5th international Emery-Dreifuss muscular dystrophy (EDMD) workshop, 1st Workshop of the MYO-CLUSTER project EUROMEN (European muscle envelope nucleopathies), 15-16 September 2000, Naarden, The Netherlands. Neuromuscul Disord. 2002;12(2):187-194.

8. Koch AJ, Holaska JM. Emerin in health and disease. Semin Cell Dev Biol. 2014;29:95-106

9. Bonne G, Quijano-Roy S. Emery-Dreifuss muscular dystrophy, laminopathies, and other nuclear envelopathies. Handb Clin Neurol. 2013;113:1367-1376.

10. Shahrizaila N, Kinnear WJM, Wills AJ. Respiratory involvement in inherited primary muscle conditions. J Neurol Neurosurg Psychiatry. 2006;77(10):1108-1115. 
11. Lerman J. Perioperative management of the paediatric patient with coexisting neuromuscular disease. Br J Anaesth. 2011;107(suppl 1): i79-i89.

12. Dickey RP, Ziter FA, Smith RA. Emery-Dreifuss muscular dystrophy. J Pediatr. 1984;104:555-559.

13. Russo V, Rago A, Palladino A, et al. P-wave duration and dispersion in patients with Emery-Dreifuss muscular dystrophy. J Invest Med. 2011;59:1151-1154.

14. Russo V, Nigro G. ICD role in preventing sudden cardiac death in EmeryDreifuss muscular dystrophy with preserved myocardial function: 2013 ESC guidelines on cardiac pacing and cardiac resynchronization therapy. Europace. 2015;17(2):337.

15. Merlini L, Granata C, Dominici P, Bonfiglioli S. Emery-Dreifuss muscular dystrophy: report of five cases in a family and review of the literature. Muscle Nerve. 1986;9:481-485.

16. Voit T, Krogmann O, Lenard HG, et al. Emery-Dreifuss muscular dystrophy: disease spectrum and differential diagnosis. Neuropediatrics. 1988;19(2):62-71.

17. Vassalle M. On the mechanisms underlying cardiac standstill: factors determining success or failure of escape pacemakers in the heart. $\mathrm{JAm}$ Coll Cardiol. 1985;5:35B-42B.

18. Gossios TD, Lopes LR, Elliott PM. Left ventricular hypertrophy caused by a novel nonsense mutation in FHL1. Eur J Med Genet. 2013;56:251-255.

19. Bonne G, Leturcq F, Ben Yaou R [webpage on the Internet]. Emery Dreifuss muscular dystrophy. In: Pagon RA, Adam MP, Ardinger HH et al., editors. GeneReviews (Internet). Seattle. University of Washington; 2004 [updated 2013]. Available from: http://www.ncbi.nlm.nih.gov/ pubmed/20301609. Accessed July 3, 2015.

20. Manilal S, Sewry CA, Man N, Muntoni F, Morris GE. Diagnosis of $\mathrm{X}$-linked Emery-Dreifuss muscular dystrophy by protein analysis of leucocytes and skin with monoclonal antibodies. Neuromuscul Disord. 1997;7(1):63-66.

21. Mora M, Cartegni L, Di Blasi C, et al. X-linked Emery-Dreifuss muscular dystrophy can be diagnosed from skin biopsy or blood sample. Ann Neurol. 1997;42(2):249-253.

22. Menezes MP, Waddell LB, Evesson FJ, et al. Importance and challenge of making an early diagnosis in LMNA-related muscular dystrophy. Neurology. 2012;78(16):1258-1263.

23. Gueneau L, Bertrand AT, Jais JP, et al. Mutations of the FHL1 gene cause Emery-Dreifuss muscular dystrophy. Am J Hum Genet. 2009;85(3):338-353.

24. Nigro V, Piluso G. Next generation sequencing (NGS) strategies for the genetic testing of myopathies. Acta Myol. 2012;31:196-200.

25. Bione S, Maestrini E, Rivella S, et al. Identification of a novel X-linked gene responsible for Emery-Dreifuss muscular dystrophy. Nat Genet. 1994;8:323-327.

26. Bonne G, Mercuri E, Muchir A, et al. Clinical and molecular genetic spectrum of autosomal dominant Emery-Dreifuss muscular dystrophy due to mutations of the lamin A/C gene. Ann Neurol. 2000;48:170-180
27. The White House [webpage on the Internet]. FACT SHEET: President Obama's Precision Medicine Initiative. Available from: www.whitehouse.gov/the-press-office/2015/01/30/fact-sheet-president-obama-sprecision-medicine-initiative. Accessed August 16, 2015.

28. Online Mendelian Inheritance in Man, OMIM ${ }^{\circledR}$ [homepage on the Internet]. Baltimore, MD: McKusick-Nathans Institute of Genetic Medicine, Johns Hopkins University. Available from: http://omim.org/. Accessed August 15, 2015.

29. Fairley EA, Kendrick-Jones J, Ellis JA. The Emery-Dreifuss muscular dystrophy phenotype arises from aberrant targeting and binding of emerin at the inner nuclear membrane. J Cell Sci. 1999;112(pt 15): 2571-2582.

30. Heibling-Leclerc A, Bonne G, Schwartz K. Emery-Dreifuss muscular dystrophy. Eur J Hum Genet. 2002;10:157-161.

31. Ellis JA, Yates JR, Kendrick-Jones J, Brown CA. Changes at P183 of emerin weaken its protein-protein interactions resulting in X-linked Emery-Dreifuss muscular dystrophy. Hum Genet. 1999;104(3):262-268.

32. Yates JR, Bagshaw J, Aksmanovic VM, et al. Genotype-phenotype analysis in X-linked Emery-Dreifuss muscular dystrophy and identification of a missense mutation associated with a milder phenotype. Neuromuscul Disord. 1999;9(3):159-165.

33. Rezania K, Pytel P, Soliven B. Chapter 23: muscle disorders. In: Gorelick PB, Testai F, Hankey G, Wardlaw JM, editors. Hankey's Clinical Neurology. 2 ed. Boca Raton, FL: CRC Press; 2014:905.

34. Rajgor D, Shanahan CM. Nesprins from the nuclear envelope and beyond. Expert Rev Mol Med. 2013;15:e5.

35. Meinke P, Mattioli E, Haque F, et al. Muscular dystrophy-associated SUN1 and SUN2 variants disrupt nuclear-cytoskeletal connections and myonuclear organization. PLoS Genet. 2014;10(9):e1004605.

36. Liang WC, Mitsuhashi H, Keduka E, et al. TMEM43 mutations in Emery-Dreifuss muscular dystrophy-related myopathy. Ann Neurol. 2011;69:1005-1013.

37. Chang W, Worman HJ, Gundersen GG. Accessorizing and anchoring the LINC complex for multifunctionality. J Cell Biol. 2015;208(1):11-22.

38. Muntoni F, Bonne G, Goldfarb LG, et al. Disease severity in dominant Emery Dreifuss is increased by mutations in both emerin and desmin proteins. Brain. 2006;129(pt 5):1260-1268

39. Ben Yaou R, Toutain A, Arimura T, et al. Multitissular involvement in a family with LMNA and EMD mutations: role of digenic mechanism? Neurology. 2007;68(22):1883-1894.

40. ClinicalTrials.gov [homepage on the Internet]. Available from: www. clinicaltrials.gov. Accessed August 10, 2015.
The Application of Clinical Genetics

\section{Publish your work in this journal}

The Application of Clinical Genetics is an international, peer-reviewed open access journal that welcomes laboratory and clinical findings in the field of human genetics. Specific topics include: Population genetics; Functional genetics; Natural history of genetic disease; Management of genetic disease; Mechanisms of genetic disease; Counseling and ethical

\section{Dovepress}

issues; Animal models; Pharmacogenetics; Prenatal diagnosis; Dysmorphology. The manuscript management system is completely online and includes a very quick and fair peer-review system, which is all easy to use. Visit http://www.dovepress.com/testimonials.php to read real quotes from published authors. 